\title{
Exercise Promotes Neurite Extensions from Grafted Dopaminergic Neurons in the Direction of the Dorsolateral Striatum in Parkinson's Disease Model Rats
}

\author{
Sadaharu Torikoshi ${ }^{\mathrm{a}, \mathrm{b}}$, Asuka Morizane ${ }^{\mathrm{a}}$, Takafumi Shimogawa ${ }^{\mathrm{a}, \mathrm{c}}$, Bumpei Samata ${ }^{\mathrm{a}}$, \\ Susumu Miyamoto ${ }^{\mathrm{b}}$ and Jun Takahashi ${ }^{\mathrm{a}, *}$ \\ ${ }^{\mathrm{a}}$ Department of Clinical Application, Center for iPS Cell Research and Application, Kyoto University, \\ Kyoto, Japan \\ ${ }^{\mathrm{b}}$ Department of Neurosurgery, Graduate School of Medicine, Kyoto University, Kyoto, Japan \\ ${ }^{\mathrm{c}}$ Department of Neurosurgery, Graduate School of Medical Sciences, Kyushu University, Fukuoka, Japan
}

Accepted 13 December 2019

\begin{abstract}
. never in PD models. and neurite extensions.

\section{INTRODUCTION}

Parkinson's disease (PD) is a neurodegenerative disorder in which a specific neural subtype dies,

\footnotetext{
*Correspondence to: Jun Takahashi, MD, PhD, Department of
} Clinical Application, Center for iPS Cell Research and Application, Kyoto University, 53 Shogoin-Kawahara-cho, Sakyo-ku, Kyoto 606-8507, Japan. Tel.: +8175366 7052; Fax.: +81 75366 7071; E-mail: jbtaka@ cira.kyoto-u.ac.jp.
\end{abstract}

Background: Cell transplantation is expected to be a promising treatment for Parkinson's disease (PD), in which reinnervation of the host striatum by grafted dopamine (DA) neurons is essential. In particular, the dorsolateral part of the striatum is important because it is the target of midbrain A9 DA neurons, which are degenerated in PD pathology. The effect of exercise on the survival and maturation of grafted neurons has been reported in several neurological disease models, but

Objective: We investigated how exercise influences cell transplantation for PD, especially from the viewpoint of cell survival

Methods: Ventral mesencephalic neurons from embryonic (E12.5) rats were transplanted into the striatum of adult 6OHDA-lesioned rats. The host rats then underwent treadmill training as exercise after the transplantation. Six weeks after the transplantation, they were sacrificed, and the grafts in the striatum were analyzed.

Results: The addition of exercise post-transplantation significantly increased the number of surviving DA neurons. Moreover, it promoted neurite extensions from the graft toward the dorsolateral part of the striatum.

Conclusions: This study indicates a beneficial effect of exercise after cell transplantation in PD.

Keywords: Parkinson's disease, cell transplantation, rehabilitation, exercise, cell survival, neuronal outgrowth

leaving patients at a loss of motor control. A9 dopamine (DA) neurons, a subtype of DA neurons, are mainly located in the substantia nigra pars compacta $(\mathrm{SNc})$ in the midbrain and are preferentially degenerated in PD. They project axons mainly to the dorsolateral part of the striatum [1]. Because the nigra-striatal pathway is involved in motor function, dopaminergic denervation to the dorsolateral striatum is significantly associated with the motor dysfunction 
in PD. In cell-based therapies, axonal outgrowth to the dorsolateral striatum from the graft is indispensable for improving PD symptoms [2-5]. Indeed, the extent of neurite distribution to the dorsolateral striatum correlates positively with behavior improvement in rat PD models [6].

In parallel with cell therapies, exercise is an established treatment for reducing the symptoms and inhibiting the disease progression of PD [7, 8]. Furthermore, several studies using toxin-induced PD model animals have shown that exercise reduces DA neuronal death and improves the behavior of the animals [9-12].

The combinatorial effect of cell transplantation and exercise has been examined in neurodegenerative diseases such as Huntington's disease [13-16] and trauma such as spinal cord injury [17, 18]. In Huntington's disease models, the animals were required to train after transplantation in order to relearn previously established habits that had been disrupted by the lesion in the striatum [15]. In studies for spinal cord injury, the survival and maturation of the grafted cells were promoted by exercise. These findings suggest that exercise enhances the function of the grafted cells. However, this combinatorial effect has never been explored in PD. In addition, its effect on axonal outgrowth, in general, remains unknown.

To address these issues, we transplanted cells obtained from rat fetal ventral mesencephalon (VM) into the striatum of PD model rats and examined the effects of exercise, especially on axonal outgrowth from the grafted DA neurons.

\section{MATERIALS AND METHODS}

\section{Preparation of fetal ventral mesencephalon}

As donor material, we used the VM dissected from the fetus of GFP-positive Sprague Dawley rats (SD-Tg (CAG-EGFP)) [19] on embryonic day 12.5. GFP staining could distinguish cell bodies and neurites derived from the graft and those from the host brain. Fjodorova and colleagues recently reported that younger (E12 vs. E14) VM donor grafts are bigger and have enhanced A9-like dopamine neuron yield [20]. VM was dissected under a microscope using a standard protocol [21]. Accumax (Innovative cell Technologies) was used to dissociate VM incubated at $37^{\circ} \mathrm{C}$ for $7 \mathrm{~min}$. The dissected VM was pipetted into single cells, washed out by PBS (-) and put on-ice during the surgery.

\section{Animals and cell transplantation}

Animals were housed according to the Guidelines for Animal Experiments of Kyoto University and Guide for the Care and Use of Laboratory Animals of the Institute of Laboratory Animal Resources (ILAR; Washington, D.C.). Adult female Sprague-Dawley rats were purchased from Shimizu Experimental Materials Co., Ltd. At 8 weeks after birth (body weight $200-250 \mathrm{~g}$ ), the median forebrain bundle of the right hemisphere of the rats was lesioned by injecting neurotoxin 6-OHDA to make the PD model. The coordinates were calculated with reference to the bregma: Anterior (A), $-4.4 \mathrm{~mm}$; Lateral (L), $-1.2 \mathrm{~mm}$; Ventral (V), $-7.8 \mathrm{~mm}$; and Tooth bar (TB), $-3.0 \mathrm{~mm}$. A total of $16 \mu \mathrm{g} 6-\mathrm{OHDA}$ was injected per rat in $2.5 \mu \mathrm{l}$ of saline with $0.02 \%$ ascorbic acid. The rats were kept under a standard environment until 39 weeks after birth, and cell transplantation was carried out at 40 weeks after birth. Cells were injected stereotactically at $1 \times 10^{5}$ cells in $2 \mu \mathrm{l}(50,000 \mathrm{cells} / \mu \mathrm{l}$, $1 \mu \mathrm{l} / 10 \mathrm{~s})$ through a $22 \mathrm{G}$ needle into the right side of the striatum (coordinates from the bregma: A, +1.0; L, -3.0; V, -5.0 and -4.0; and TB, -2.0). Six weeks after the cell transplantation, the animals were euthanized with pentobarbital and perfused with $4 \%$ paraformaldehyde. The brains were cut at $50 \mu \mathrm{m}$ thickness using a cryostat and mounted.

\section{Experimental design}

Twenty-four PD model rats ran on a treadmill machine for 5 consecutive days $15 \mathrm{~min}$ a day (velocity $=15 \mathrm{~cm} / \mathrm{s}$ for $10 \mathrm{~min}$ followed by $20 \mathrm{~cm} / \mathrm{s}$ for $5 \mathrm{~min}$ ) as pre-training 4 weeks prior to the cell transplantation (Fig. 1C). The treadmill machine has five lanes with metal plates at the rear through which electricity $(0.2 \mathrm{~mA})$ flows when the hind legs come into contact with the plate to motivate the rats to keep running (Treadmill machine; Harvard Apparatus, MA, USA) (Fig. 1D). Overall, based on the pre-rotation test performed 5 weeks prior to the cell transplantation, the 24 PD model rats were divided into four equally sized groups so that the average number of rotations in each group were almost the same: transplantation (Tx), TMT, Tx+TMT, and control group. Rats in the Tx group underwent just cell transplantation. Rats in the TMT group received $2 \mu$ injection of saline and afterward did the treadmill running. Rats in the Tx+TMT group did the treadmill running after receiving the cell transplantation. Rats in the control group received only $2 \mu l$ injection of saline. The 
A Preparation of donor cells

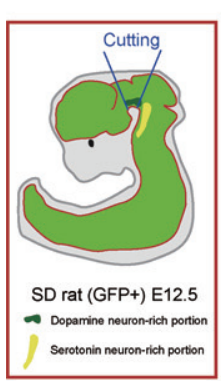

Cutting

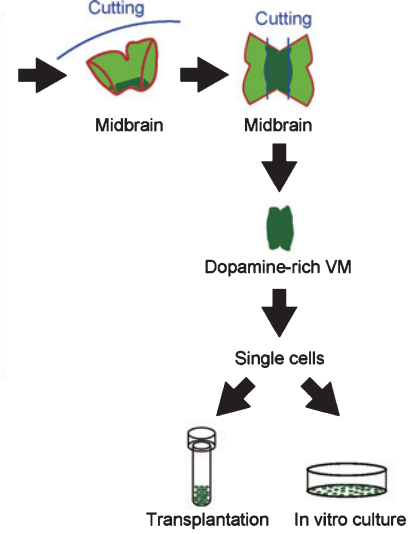

B SD rat (GFP+) E12.5 Ventral mesencephlon 6 days culture in vitro

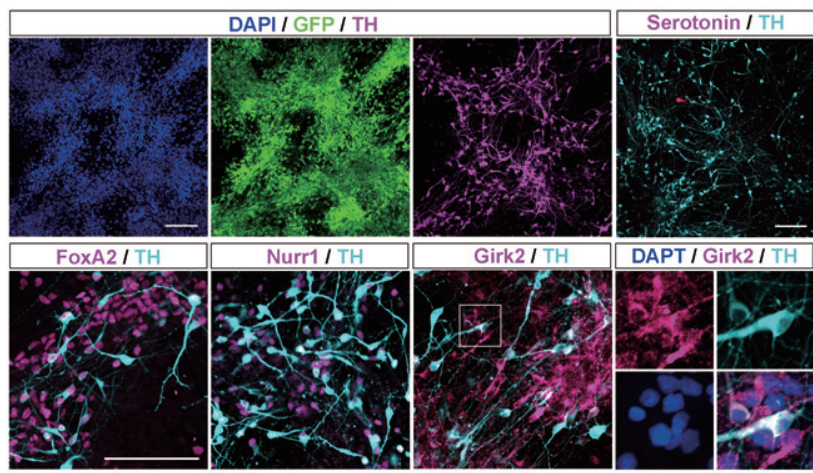

C
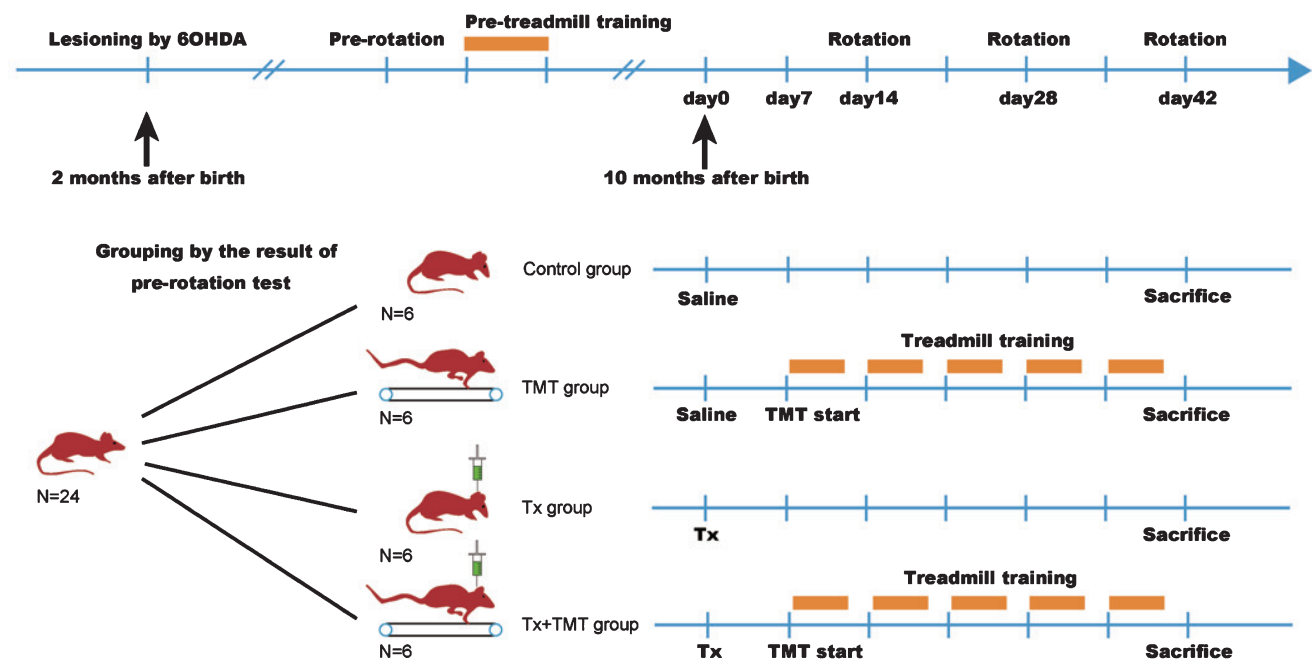

$\mathrm{D}$

E

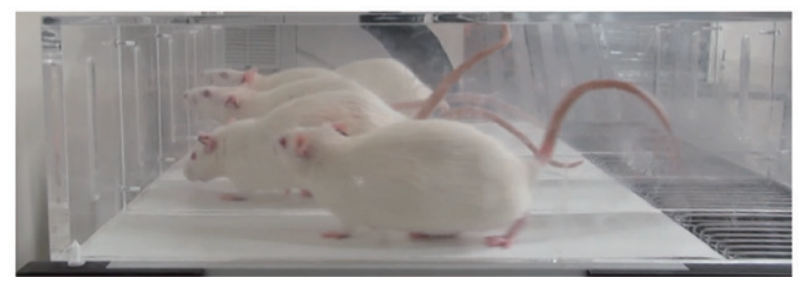

\section{Treadmill training schedule}

$30 \mathrm{~min} /$ day, 5 days/week for 5 weeks

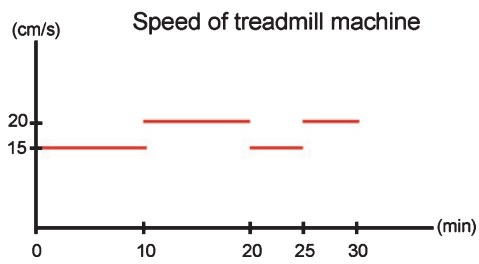

Fig. 1. Donor cell preparation and experimental design. (A) Dissection of ventral mesencephalon (VM) from GFP-positive Sprague-Dawley (SD) rat (E12.5). The rostral side of the VM was dissected so as not to include serotoninergic neurons. (B) Immunocytological evaluation of donor cells cultured in vitro on a poly-L-O/F/L-coated chamber slide for 6 days. (C) Experimental design. Adult chronic Parkinson's disease model rats $(\mathrm{N}=24)$ were divided into 4 groups: Tx, TMT, Tx+TMT, and Control. (D) A picture of rats running on the treadmill machine. (E) Treadmill training protocol. The velocity of the treadmill was as follows: $15 \mathrm{~cm} / \mathrm{s}$ for 10 minutes, $20 \mathrm{~cm} / \mathrm{s}$ for $10 \mathrm{~min}, 15 \mathrm{~cm} / \mathrm{s}$ for $5 \mathrm{mins}$, and $20 \mathrm{~cm} / \mathrm{s}$ for $5 \mathrm{~min}$ (total $30 \mathrm{~min}$ ). Tx, only cell transplantation; TMT, saline injection and treadmill training; Tx+TMT, cell transplantation and treadmill training; Control, only saline injection; Rotation, amphetamine-induced rotational behavior test. Scale bars: $100 \mu \mathrm{m}$. 
amphetamine-induced rotation test was performed on days 14,28 , and 42 after the transplantation. The protocol of the treadmill training was described previously [22]. Treadmill training started 7 days after the transplantation, 30 min per day, 5 days per week for 5 continuous weeks. According to some reports, the effect of treadmill training depends on the running velocity [23]. In the present study, the velocity was changed four times during the $30 \mathrm{~min}$-treadmill training $(15 \mathrm{~cm} / \mathrm{s}$ for $10 \mathrm{~min}, 20 \mathrm{~cm} / \mathrm{s}$ for $10 \mathrm{~min} 15 \mathrm{~cm} / \mathrm{s}$ for $5 \mathrm{~min}$, and $20 \mathrm{~cm} / \mathrm{s}$ for $5 \mathrm{~min}$ ) (Fig. 1E). The rats were sacrificed at six weeks after the transplantation according to a previous report [24].

\section{Behavior analysis}

The amphetamine-induced rotation test was performed to evaluate behavioral recovery at pre-transplantation and every 2 weeks after transplantation using a rotameter apparatus (RotoRat; Med Associates Inc). A dose of $2.5 \mathrm{mg} / \mathrm{kg}$ methamphetamine (Dainippon Sumitomo Pharma) was administered intraperitoneally, and the number of rotations was automatically recorded for $90 \mathrm{~min}$. Only the rats that exhibited more than 6 rotations per minute were entered in this study as PD models.

\section{Immunohistochemistry}

Immunohistochemical analyses were performed after permeabilization and blocking with $0.3 \%$ Triton X-100 and $2 \%$ skim milk. Immunoreactive cells were observed by using a fluorescence microscope (BZ-9000; Keyence) and a confocal laser microscope (LSM 700; Zeiss). The primary antibodies used were goat anti-FOXA2 (1:500, Santa Cruz Biotechnology), rabbit anti-GIRK2 (1:200, Alomone Labs), rabbit anti-TH (1:400, Millipore), rat anti-GFP (1:1000, Nacalaitesque), rat anti-NURR1 (1:1000, KAN research institute), rat anti-SEROTONIN (1:100, Millipore), and sheep anti-TH (1:200, Millipore).

\section{Quantification}

The brains were cut at $50 \mu \mathrm{m}$ thickness, and the striatum containing a graft was divided into six sets of 12 slices. The number of neurons positive for TH or Girk2 in a graft were counted manually through all graft images of each rat captured by a fluorescence microscope (BZ-9000; Keyence). Graft volume $\left(\mathrm{mm}^{3}\right)$ and graft surface area $\left(\mathrm{mm}^{2}\right)$ were calculated by measuring the GFP-positive area of the graft. Neurites double-positive for GFP and Girk2 were derived from the grafted cells (Fig. 3C). We adopted Girk2 to quantify neurites because of its strong contrast and excellent quantification compared to GFP. Girk2-positive neurites extending from grafted dopaminergic neurons were traced manually and calculated into total neurite length $(\mathrm{mm})$ by using the BZ-9000 Keyence work station. To examine which direction the neurites extended from the graft, the striatum was divided into four areas around the graft: dorsolateral, ventrolateral, dorsomedial, and ventromedial striatum, and total neurite length was calculated and quantified in each area. In addition, to examine how far the neurites from the graft edge reached in the direction of the dorsolateral striatum, contour lines were drawn from the graft edge to a maximum of $500 \mu \mathrm{m}$ in $100 \mu \mathrm{m}$ increments, and the number of axons passing through those contours was counted. Imaging analyses were performed with the software Photoshop CC 2018.

\section{Statistical analysis}

Statistical analysis was performed using a commercially available software package (GraphPad Prism 7; GraphPad Software). Data obtained from the in vivo experiments were analyzed by the Unpaired $t$ test or Mann-Whitney test. Behavior assessments were analyzed with a two-way ANOVA with Dunnett's multiple comparisons test. The differences were considered to be statistically significant if $p<0.05$. The data are presented as the mean $\pm \mathrm{SD}$ or mean \pm SEM.

\section{RESULTS}

\section{Exercise improves survival of grafted DA neurons}

To obtain donor cells, VM tissue was dissected from the fetus of GFP-positive Sprague Dawley rats on embryonic day 12.5 (E12.5) [20]. The VM tissue was dissociated and subjected to in vitro culture and transplantation (Fig. 1A). An immunofluorescence study of the cultured VM tissue showed that all cells expressed GFP and some expressed DA neuron markers such as Forkhead box protein A2 (FOXA2), Nuclear receptor related 1 protein (NURR1) and tyrosine hydroxylase (TH). Moreover, most $\mathrm{TH}+$ cells expressed G-protein-regulated inward-rectifier potassium channel 2 (Girk2) (Fig. 1B). Because we 

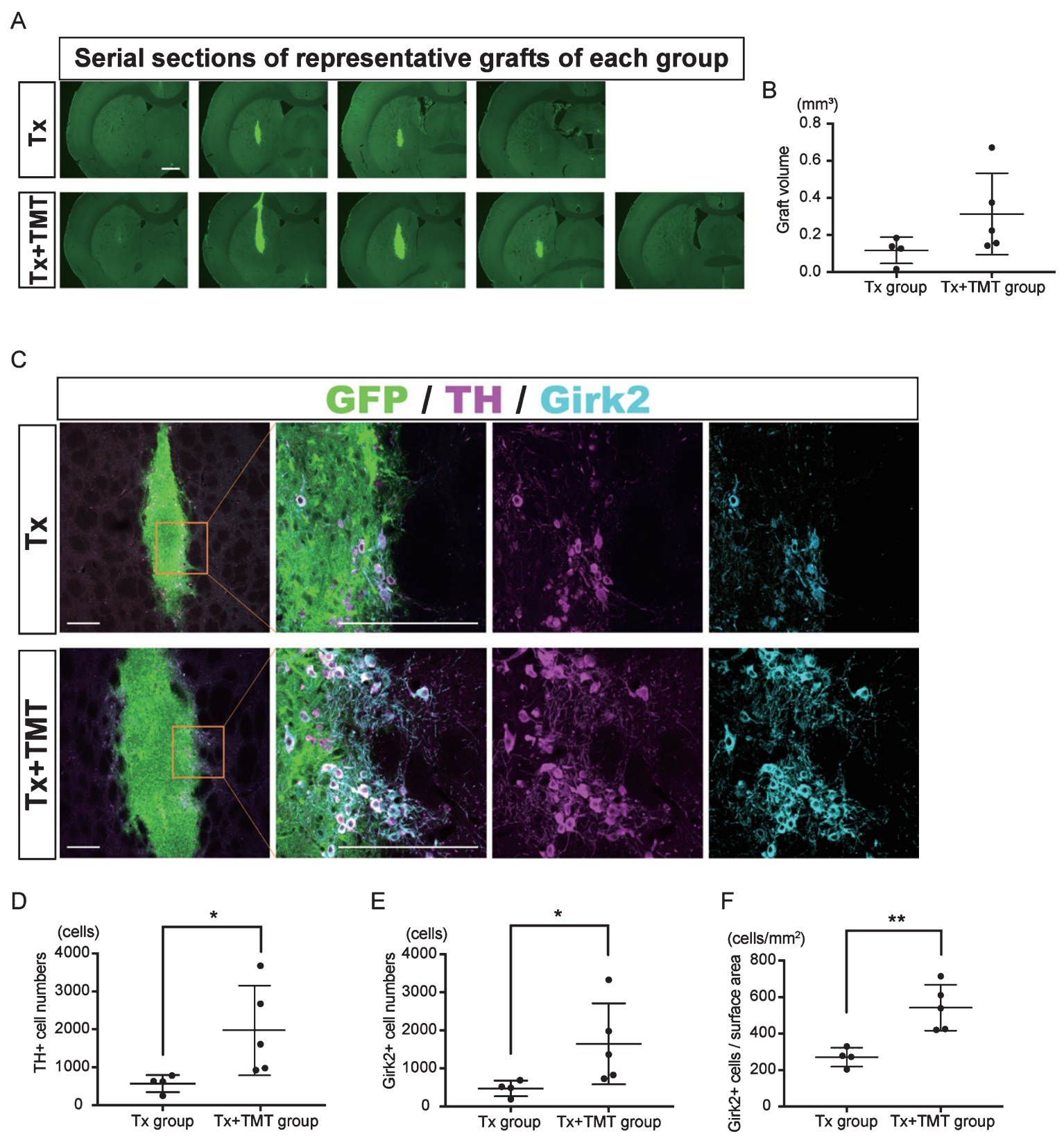

Fig. 2. Exercise increased the number of dopamine neurons in the graft. (A) Serial sections of representative grafts at 6 weeks after transplantation in Tx and Tx+TMT rats. The grafts were identified with the expression of GFP. (B) Quantification of the graft volume from the Tx group $(\mathrm{N}=4)$ and Tx+TMT group $(\mathrm{N}=5)$. (C) Immunofluorescence images of the grafts. (D, E and F) Quantification of TH+ (D) and Girk2+ (E, F) cells in the grafts. The number of TH-positive cells was significantly larger in the Tx+TMT group (D). More Girk2-positive cells survived in the Tx+TMT group, both in total number (E) and in number adjusted by the surface area of the graft $\left(\mathrm{cell}_{\mathrm{s}} / \mathrm{cm}^{2}, \mathrm{~F}\right) .{ }^{*} p=0.016$ by Mann-Whitney test, ${ }^{* *} p=0.005$ by Unpaired $t$ test. All values are expressed as the mean \pm SD (B, D, E and F). Scale bars: 1 mm (A), $200 \mu \mathrm{m}(\mathrm{C})$.

dissected carefully to avoid hindbrain tissue, only few serotonergic neurons were observed.

Two-month-old SD rats were treated with 6hydroxydopamine and underwent cell transplantation eight months later. An immunohistochemical study at six-weeks post-transplantation demonstrated graft survival in four out of six rats with transplantation alone (Tx) and five out of six rats with transplantation and exercise (Tx+TMT) (Fig. 2A, Supplementary Figure 1). The grafted cells and their neurites 
A
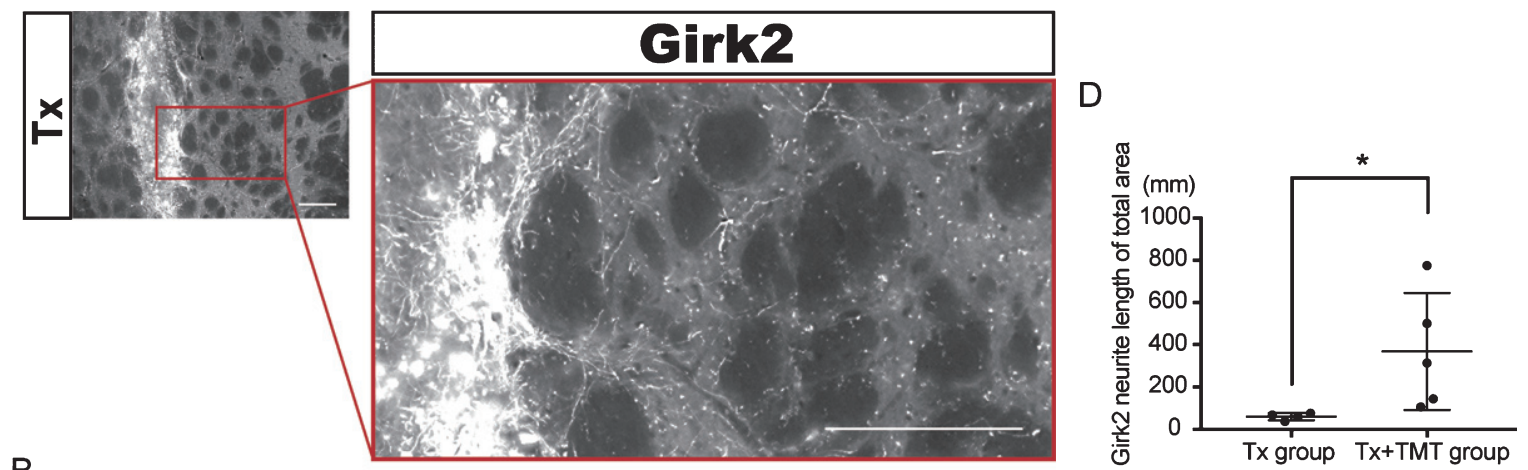

B

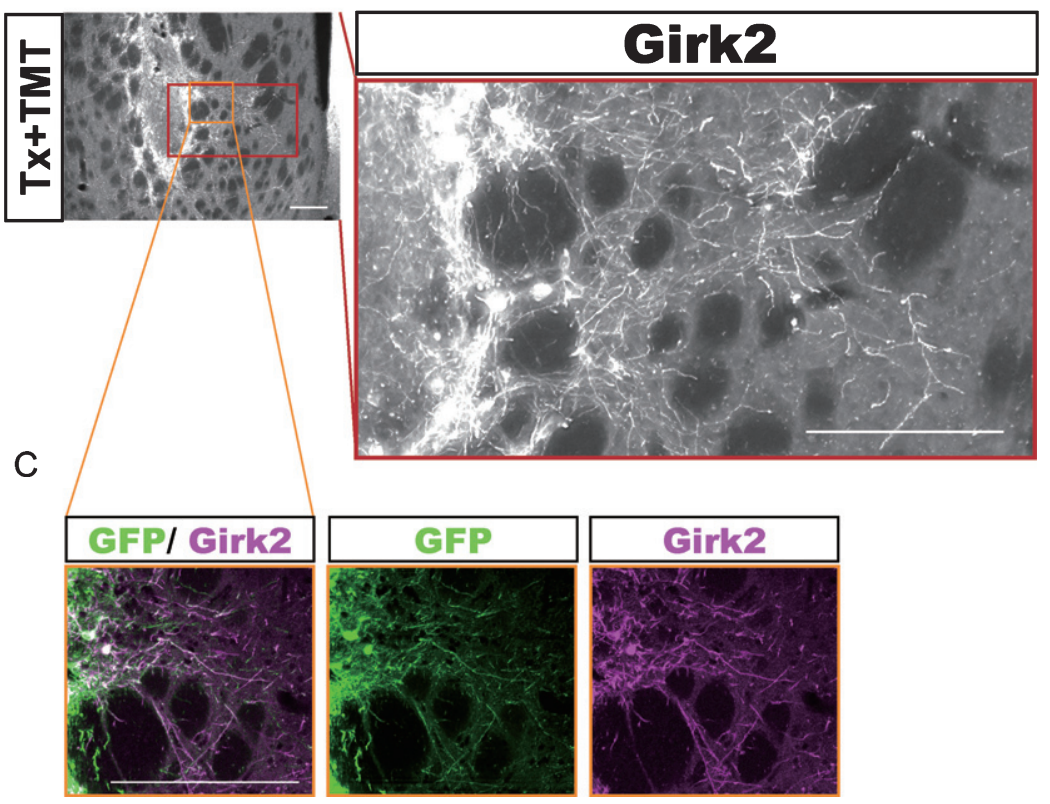

$E$

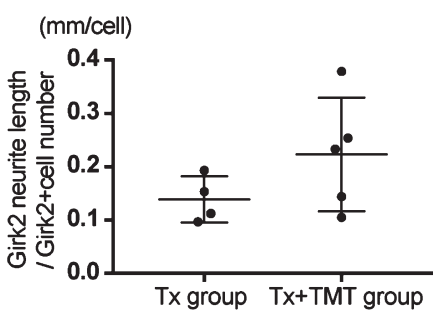

Fig. 3. Exercise promoted neurite outgrowth from the graft. (A-C) Representative immunofluorescence images of Girk2-positive neurites extended from the graft in Tx (A) and Tx+TMT (B, C) groups. All Girk2-positive neurites expressed GFP (C). Scale bars: $200 \mu \mathrm{m}$. (D, E) Quantification of Girk2-positive neurites extended from the graft. Girk2-positive neurites were traced manually and quantified into total neurite length (D). * $p=0.041$ by Mann-Whitney test. (E) Adjusted length of Girk2-positive neurites by the number of Girk2-positive cells. $p=0.1864$ by Unpaired $t$ test.

were histologically identified by the GFP signal (Fig. 2A-C, Fig. 3C). There was no significant difference in the graft volume between the Tx and Tx+TMT groups $\left(0.1171 \pm 0.071\right.$ and $0.314 \pm 0.2198 \mathrm{~mm}^{3}$, respectively. $p=0.1327$ ) (Fig. $2 \mathrm{~B}$ ). In the peripheral area of the graft, the surviving cells were large and angular in shape, and expressed both $\mathrm{TH}$ and Girk2 (Fig. 2C, Supplementary Figure 1B, D). On the contrary, small and rounded TH positive cells were scattered in the core of the graft, and they did not express Girk2, as shown previously [25]. The number of TH-positive cells per graft was significantly larger in the Tx+TMT group than in the Tx group $(1970 \pm 1183$ and $571.5 \pm 224.8$ cells, respectively. $p=0.016$ ) (Fig. 2D). Similarly, the number of Girk2-positive cells was significantly larger in the Tx+TMT group than in the Tx group $(1648 \pm 1064$ and $474 \pm 207.2$ cells, respectively. $p=0.016$ ) (Fig. 2E). The number of surviving THpositive cells was relatively few compared to that of a previous report [26], which we attributed to the old age (40 weeks) of the host rats in this study [27, 28]. Since most of the $\mathrm{TH}+/$ Girk2+ cells were distributed on the graft surface, the number of Girk2+ cells per surface area of the graft, namely, the density of midbrain DA neurons, was calculated. This value was 
also significantly larger in the Tx+TMT group than in the Tx group $(542.5 \pm 125.3$ and $271.5 \pm 52.10$ cell $/ \mathrm{mm}^{2}$, respectively. $p=0.005$ ) (Fig. $2 \mathrm{~F}$ ), indicating that exercise improved the survival of the grafted DA neurons. At the same time, the ratio of Girk2positive cells to TH-positive cells was about $80 \%$ in both groups $(0.829 \pm 0.0793$ and $0.816 \pm 0.0534$, respectively), indicating that exercise did not alter the ratio of Girk2+ DA neurons to total DA neurons among the surviving DA neurons.

\section{Exercise promotes neurite extensions in the direction of the dorsolateral striatum}

Next, Girk2-positive neurites from the grafts were traced manually, and their total length was measured (Fig. 3A-C). The neurite length in the whole striatum was significantly longer in the Tx+TMT group than in the Tx group $(367.9 \pm 276.4$ and $59.51 \pm 16.42 \mathrm{~mm}$, respectively. $p=0.016$ ) (Fig. 3D). However, there was no significant difference in the neurite length per cell between the two groups $(0.223 \pm 0.106$ and $0.139 \pm 0.043 \mathrm{~mm} /$ cell, respectively. $p=0.186$ ) (Fig. 3E). These results suggested that the increase in total neurite length in the Tx+TMT group was because of the increased number of surviving DA neurons in the graft. However, exercise could have affected the directional diversity of the neurite extensions from the grafted neurons in the striatum. Therefore, we conducted a finer analysis, in which we divided the striatum into four regions and measured the neurite length in each region. As a result, we found that in the dorsolateral striatum, the total neurite length was significantly increased in the Tx+TMT group compared with the Tx group $(48.52 \pm 35.37$ and $6.894 \pm 3.72 \mathrm{~mm}$, respectively. $p=0.016$ ) (Fig. 4A-C). Furthermore, the average neurite length of each Girk2+ DA neuron in the dorsolateral striatum was also significantly increased by exercise $(0.116 \pm 0.031$ and $0.056 \pm 0.007 \mathrm{~mm} / \mathrm{cell}$, respectively. $p=0.016$ ) (Fig. 4D). To confirm the enhanced neurite extensions in the direction of the dorsolateral striatum by exercise, we counted the number of neurites that reached 100 to $500 \mu \mathrm{m}$ dorsolaterally from the graft edge (Fig. 4E). Again, the total number of Girk2+ neurites and the number of neurites from each Girk2+ DA neuron were significantly increased in the Tx+TMT group (Fig. 4F, G). Intriguingly, the other three directions did not show an increase in neurite length per cell (Fig. 4H-J). These results suggested that exercise after cell transplantation selectively enhances neurite extensions from the grafted Girk2+ DA neurons to the direction of the dorsolateral striatum.

Neither Girk2+ neurites nor GFP+ neurites were found in the cortex, and neurite extensions were confined to the striatum. This result is different from that of previous reports $[25,29,30]$, which we attribute to the host brain environment of our aged rats difficult in obtaining neurite extensions [27].

\section{Behavioral improvement was correlated with neurite extensions to the dorsolateral striatum}

The behavior of rats following cell transplantation was evaluated by an amphetamine-induced rotation test. Both Tx and Tx+TMT groups showed a complete reduction in rotations at 4 and 6 weeks after transplantation, indicating that the grafted cells functioned as DA neurons (Fig. 5A, and Supplementary Table 1). No significant difference was observed between the two groups, but both of them showed a significant reduction in rotations compared to the control group. Additionally, behavioral improvement was not observed in the TMT group (exercise only), consistent with a previous report describing 6-OHDA-lesioned rats [12]. The improvement ratio by the rotation test showed a correlation with neurite extensions to the dorsolateral striatum $\left[R^{2}=0.6249\right]$, and the data suggested that even the smallest of neurite extensions $(2.309 \mathrm{~mm})$ was enough to exert almost full recovery (Fig. 5B).

\section{DISCUSSION}

In this study, we grafted the ventral midbrain of E12.5 rats to the striatum of 6-OHDA-leioned rats and found that exercise post-transplantation promotes the survival and maturation of the grafted DA neurons. The exercise also enhanced the neurite extensions from the grafts into the dorsolateral striatum (Supplementary Figure 2). In the midbrain, two types of DA neurons exist: A9 DA neurons, which innervate the striatum, and A10 DA neurons, which innervate the frontal cortex. When grafted in the striatum, only A9 DA neurons show robust axonal outgrowth in the striatum and cause behavioral improvement $[25,31$, 32]. Our immunofluorescence studies revealed that the number of midbrain DA neurons (TH+/Girk2+) was increased by exercise. In addition, intriguingly, the neurite length per cell was significantly increased only in the direction of the dorsolateral striatum, which is the main target of physiological A9 DA 
A

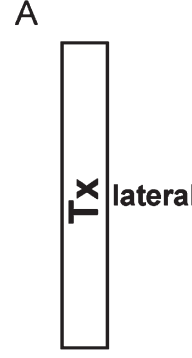

B

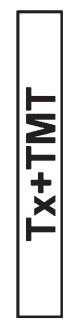

dorsal

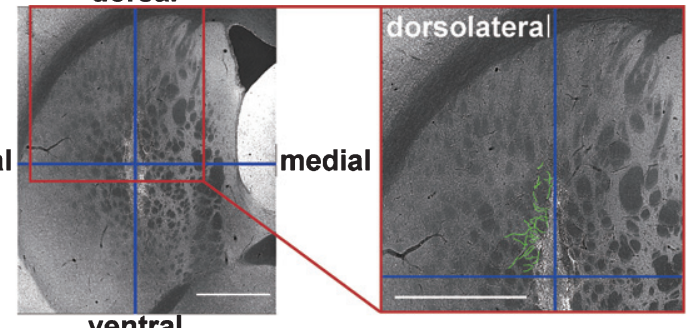

ventral

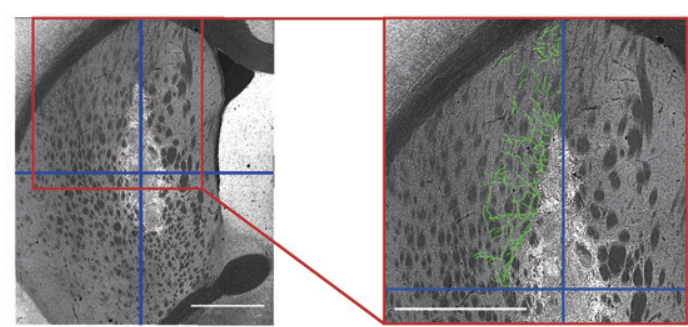

C

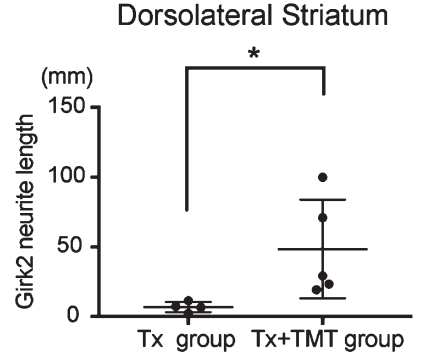

D

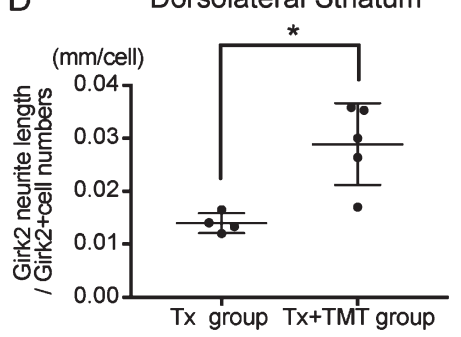

G
E

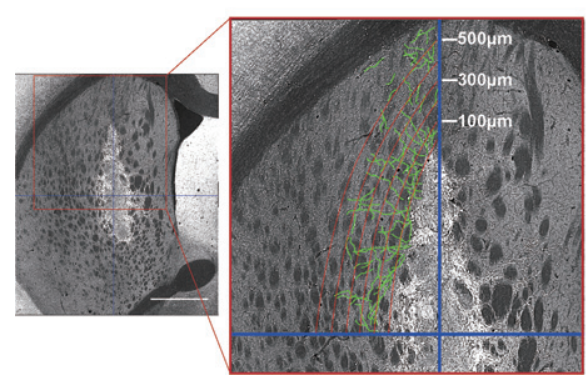

F

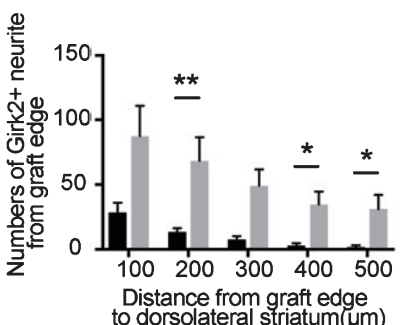

H Ventrolateral Striatum

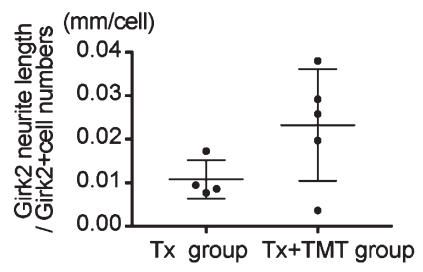

I
Dorsomedial Striatum

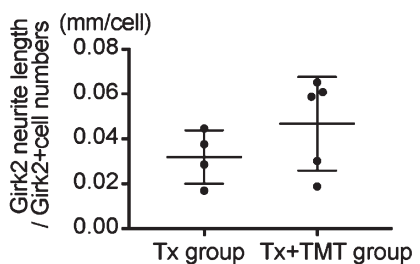

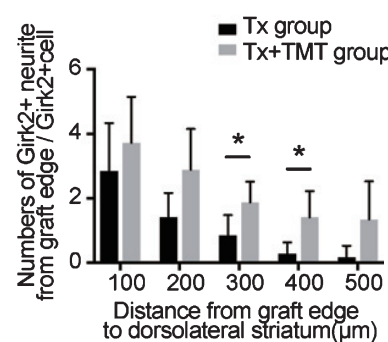

J Ventromedial Striatum

Fig. 4. Exercise enhanced neurite outgrowth in the direction of the dorsolateral striatum. (A, B) To estimate the direction of neurite outgrowth, the striatum was divided into 4 regions around the grafts. Division lines (blue) in the striatum are indicated with representative images of Girk2 staining from the Tx group (A) and Tx+TMT group (B). Girk2-positive neurites from the graft in each area were traced manually (green lines), and their total length was measured. Scale bars: $1 \mathrm{~mm}$. (C) Quantification of Girk2-positive total neurite length in the dorsolateral striatum. ${ }^{*} p=0.016$ by Mann-Whitney test. (D) Quantification of the adjusted Girk2-positive neurite length per Girk2-positive unit cell number in the dorsolateral striatum. ${ }^{*} p=0.016$ by Mann-Whitney test. (E) Estimation of the reaching distance of Girk2-positive neurites from the graft edge in the dorsolateral striatum. Contour lines were drawn from the graft edge to $500 \mu \mathrm{m}$ in $100 \mu \mathrm{m}$ increments, and the number of neurites passing through those contours were counted. Scale bar: $1 \mathrm{~mm}$. These lines were used for the bar graphs in (F) and (G). (F) Number of Girk2-positive neurites from the graft edge passing through each contour. $200 \mu \mathrm{m}: * * p=0.008,400 \mu \mathrm{m}: * p=0.032,500 \mu \mathrm{m}$ : ${ }^{*} p=0.024$ by Mann-Whitney test. (G) Number of Girk2-positive neurites per unit cell number from the graft edge passing through each contour. $300 \mu \mathrm{m}: * p=0.049,400 \mu \mathrm{m}: * p=0.036$ by Unpaired $t$ test. $(\mathrm{H}-\mathrm{J})$ Girk2-positive neurite length per unit cell number in each area. Ventrolateral striatum: $P=0.1077(\mathrm{H})$, dorsomedial striatum: $p=0.2498(\mathrm{I})$, and ventromedial striatum: $p=0.1905(\mathrm{~J})$. Values are expressed as the mean $\pm \mathrm{SD}(\mathrm{C}, \mathrm{D}, \mathrm{F}-\mathrm{J})$. Tx group, transplantation group; Tx+TMT group, transplantation with treadmill training group.

neuronal projections [1]. These results suggest that exercise can promote the therapeutic effect of the grafted DA neurons in the striatum of a PD model.
According to previous reports, the mechanism of the exercise effect is speculated as follows. In rodents, glutamatergic neurons in the cortical motor 

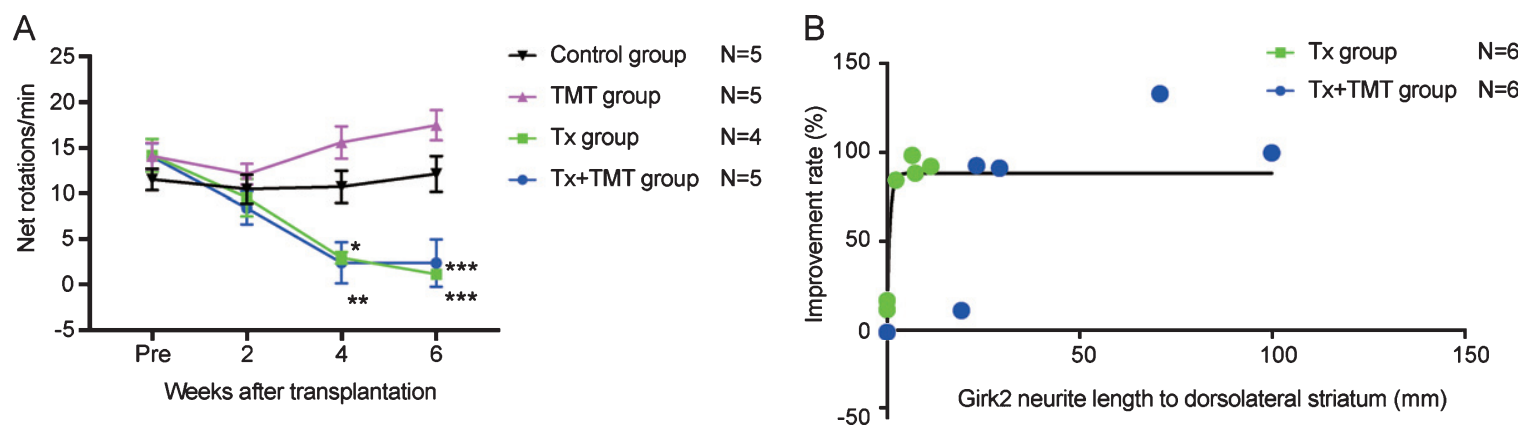

Fig. 5. Behavior analysis by amphetamine-induced rotation test. (A) Amphetamine-induced rotation test. Values are expressed as the mean \pm SEM. $* p=0.015$ (Tx group, 4 weeks), $* * p=0.005$ (Tx+TMT group, 4 weeks), $* * * p<0.001$ (Tx group and Tx + TMT group, 6 weeks) by two-way ANOVA with Tukey's multiple comparisons test. (B) Relationship between Girk2-positive neurites in the direction of the dorsolateral striatum and improvement rate of the rotation test in Tx and Tx+TMT groups. $\mathrm{R}^{2}=0.6249$ by non-linear regression.

area, which form a cortico-striatal pathway, innervate medium spiny neurons (MSNs) in the dorsolateral striatum [33]. Exercise promotes the release of glutamates from glutamatergic nerve terminals in the dorsolateral striatum [34] and up-regulates glutamate receptors on MSNs [35]. Exercise also increases the number of dendritic spines on MSNs [36]. On the other hand, DA release from DA neurons supports the firing of MSNs in the striatum excited by glutamatergic neurons in the motor cortex [37]. These findings suggest that exercise enhances the activities and requirement of DA by MSNs in the dorsolateral striatum, which lures axons of the grafted DA neurons.

It should be noted that the rats we used are older than those reported in previous studies. There were several reasons for this decision. One is because a majority of PD patients are older. In addition, some studies have shown recovery or protective effects of exercise at the acute phase of the 6-OHDA lesioning in rats $[9,10,12]$. Therefore, to avoid these recovery and protective effects, we waited a long time ( 8 months) between the lesioning and transplantation to investigate the exercise effect on the grafts. Consequently, our TMT (exercise only) group showed no behavioral improvement.

This study is, to our knowledge, the first report to confirm the interaction between cell-based therapy and exercise in a PD model. In stroke [38] and spinal cord injury models $[17,18]$, it has been reported that exercise principally promotes the survival and maturation of the grafted cells. Consistent with these previous studies, we found exercise improved survival of the grafted DA neurons. Because a low survival rate of the grafted cells is one of the main problems limiting cell-based therapies for PD [39,
40], the effect of exercise should benefit PD cell therapies. Several mechanisms have been reported about the effects of exercise on cell survival and maturation, including the up-regulation of neurotrophic factors such as BDNF and GDNF [41-43] and the modulation of inflammatory processes through the reduction of inflammatory cytokines, which minimizes the mitochondrial dysfunction of DA neurons $[11,22]$. Similar mechanisms might support the survival and neurite extensions of grafted DA neurons in the striatum.

In this study, exercise after cell transplantation had no influence on rat behavior in an amphetamineinduced rotation test despite the enhanced cell survival and axonal outgrowth. It is presumed that the grafted DA neurons secreted sufficient amounts of DA to ameliorate amphetamine-induced rotations even without exercise, resulting in a saturated effect. Therefore, the enhanced survival and neurite extensions of the grafted DA neurons and the altered axonal distribution due to exercise did not contribute to the behavioral improvement assessed by this test. To show the effect of exercise after cell transplantation on behavioral improvement, other behavioral analyses including the apomorphine induced rotation test, cylinder test, corridor test, and others would be helpful. Because these tests are able to detect the fine functional changes of PD models previously [24], they are recommended to study the effects of exercise on the grafts.

Exercise will almost certainly be applied as part of the rehabilitation after a cell transplantation for the treatment of PD. This study shows the beneficial effects of exercise conducted after the transplantation in rats. 


\section{ACKNOWLEDGMENTS}

We thank Dr. Peter Karagiannis for critical reading of the manuscript, Dr. Tetsuhiro Kikuchi for technical advice on the quantification of neurite length, and Dr. Takenori Ogura and Dr. Yulius Hermanto for their technical assistance.

This work was supported by a grant from the Network Program for Realization of Regenerative Medicine from the Japan Agency for Medical Research and Development (AMED) (18bm0204004h0006 to J.T.).

\section{CONFLICT OF INTEREST}

The authors have no conflict of interest to report.

\section{SUPPLEMENTARY MATERIAL}

The supplementary material is available in the electronic version of this article: https://dx.doi.org/10. 3233/JPD-191755.

\section{REFERENCES}

[1] Bjorklund A, Dunnett SB (2007) Dopamine neuron systems in the brain: An update. Trends Neurosci 30, 194-202.

[2] Wictorin K, Brundin P, Sauer H, Lindvall O, Bjorklund A (1992) Long distance directed axonal growth from human dopaminergic mesencephalic neuroblasts implanted along the nigrostriatal pathway in 6-hydroxydopamine lesioned adult rats. J Comp Neurol 323, 475-494.

[3] Thompson LH, Grealish S, Kirik D, Bjorklund A (2009) Reconstruction of the nigrostriatal dopamine pathway in the adult mouse brain. Eur J Neurosci 30, 625-638.

[4] Kirkeby A, Grealish S, Wolf DA, Nelander J, Wood J, Lundblad M, Lindvall O, Parmar M (2012) Generation of regionally specified neural progenitors and functional neurons from human embryonic stem cells under defined conditions. Cell Rep 1, 703-714.

[5] Grealish S, Diguet E, Kirkeby A, Mattsson B, Heuer A, Bramoulle Y, Van Camp N, Perrier AL, Hantraye P, Bjorklund A, Parmar M (2014) Human ESC-derived dopamine neurons show similar preclinical efficacy and potency to fetal neurons when grafted in a rat model of Parkinson's disease. Cell Stem Cell 15, 653-665.

[6] Cardoso T, Adler A, Mattsson B, Hoban DB, Nolbrant S, Wahlestedt JN, Kirkeby A, Grealish S, Bjorklund A, Parmar M (2018) Target-specific forebrain projections and appropriate synaptic inputs of hESC-derived dopamine neurons grafted to the midbrain of parkinsonian rats. J Comp Neurol 526, 2133-2146.

[7] Tomlinson CL, Patel S, Meek C, Herd CP, Clarke CE, Stowe R, Shah L, Sackley C, Deane KH, Wheatley K, Ives N (2012) Physiotherapy intervention in Parkinson's disease: Systematic review and meta-analysis. BMJ 345, e5004.
[8] Bloem BR, de Vries NM, Ebersbach G (2015) Nonpharmacological treatments for patients with Parkinson's disease. Mov Disord 30, 1504-1520.

[9] Tillerson J, Caudle W, Reveron M, Miller G (2003) Exercise induces behavioral recovery and attenuates neurochemical deficits in rodent models of Parkinson's disease. Neuroscience 119, 899-911.

[10] Yoon M-C, Shin M-S, Kim T-S, Kim B-K, Ko I-G, Sung YH, Kim S-E, Lee H-H, Kim Y-P, Kim C-J (2007) Treadmill exercise suppresses nigrostriatal dopaminergic neuronal loss in 6-hydroxydopamine-induced Parkinson's rats. $\mathrm{Neu}$ rosci Lett 423, 12-17.

[11] Lau YS, Patki G, Das-Panja K, Le WD, Ahmad SO (2011) Neuroprotective effects and mechanisms of exercise in a chronic mouse model of Parkinson's disease with moderate neurodegeneration. Eur J Neurosci 33, 1264-1274.

[12] Petzinger GM, Fisher BE, McEwen S, Beeler JA, Walsh JP, Jakowec MW (2013) Exercise-enhanced neuroplasticity targeting motor and cognitive circuitry in Parkinson's disease. Lancet Neurol 12, 716-726.

[13] Brasted PJ, Watts C, Torres EM, Robbins TW, Dunnett SB (2000) Behavioral recovery after transplantation into a rat model of Huntington's disease: Dependence on anatomical connectivity and extensive postoperative training. Behav Neurosci 114, 431-436.

[14] Brasted PJ, Watts C, Torres EM, Robbins TW, Dunnett SB (1999) Behavioural recovery following striatal transplantation: Effects of postoperative training and P-zone volume. Exp Brain Res 128, 535-538.

[15] Brasted PJ, Watts C, Robbins TW, Dunnett SB (1999) Associative plasticity in striatal transplants. Proc Natl Acad Sci U S A 96, 10524-10529.

[16] Mayer E, Brown VJ, Dunnett SB, Robbins TW (1992) Striatal graft-associated recovery of a lesion-induced performance deficit in the rat requires learning to use the transplant. Eur J Neurosci 4, 119-126.

[17] Hwang DH, Shin HY, Kwon MJ, Choi JY, Ryu BY, Kim BG (2014) Survival of neural stem cell grafts in the lesioned spinal cord is enhanced by a combination of treadmill locomotor training via insulin-like growth factor-1 signaling. $J$ Neurosci 34, 12788-12800.

[18] Tashiro S, Nishimura S, Iwai H, Sugai K, Zhang L, Shinozaki M, Iwanami A, Toyama Y, Liu M, Okano H, Nakamura M (2016) Functional recovery from neural stem/progenitor cell transplantation combined with treadmill training in mice with chronic spinal cord injury. $S c i$ Rep 6, 30898 .

[19] Ito T, Suzuki A, Imai E, Okabe M, Hori M (2001) Bone marrow is a reservoir of repopulating mesangial cells during glomerular remodeling. J Am Soc Nephrol 12, 2625-2635.

[20] Fjodorova M, Torres EM, Dunnett SB (2017) Transplantation site influences the phenotypic differentiation of dopamine neurons in ventral mesencephalic grafts in Parkinsonian rats. Exp Neurol 291, 8-19.

[21] Dunnett SB, Bjorklund A (1997) Basic neural transplantation techniques. I. Dissociated cell suspension grafts of embryonic ventral mesencephalon in the adult rat brain. Brain Res Brain Res Protoc 1, 91-99.

[22] Svensson M, Lexell J, Deierborg T (2015) Effects of physical exercise on neuroinflammation, neuroplasticity, neurodegeneration, and behavior: What we can learn from animal models in clinical settings. Neurorehabil Neural Repair 29, 577-589.

[23] Funato T, Yamamoto Y, Aoi S, Imai T, Aoyagi T, Tomita N, Tsuchiya K (2016) Evaluation of the phase-dependent 
rhythm control of human walking using phase response curves. PLoS Comput Biol 12, e1004950.

[24] Torres EM, Dowd E, Dunnett SB (2008) Recovery of functional deficits following early donor age ventral mesencephalic grafts in a rat model of Parkinson's disease. Neuroscience 154, 631-640.

[25] Thompson L, Barraud P, Andersson E, Kirik D, Bjorklund A (2005) Identification of dopaminergic neurons of nigral and ventral tegmental area subtypes in grafts of fetal ventral mesencephalon based on cell morphology, protein expression, and efferent projections. $J$ Neurosci 25, 6467-6477.

[26] Nakao N, Frodl EM, Duan WM, Widner H, Brundin P (1994) Lazaroids improve the survival of grafted rat embryonic dopamine neurons. Proc Natl Acad Sci U S A 91, 12408-12412.

[27] Collier TJ, Sortwell CE, Daley BF (1999) Diminished viability, growth, and behavioral efficacy of fetal dopamine neuron grafts in aging rats with long-term dopamine depletion: An argument for neurotrophic supplementation. $J$ Neurosci 19, 5563-5573.

[28] Collier TJ, O'Malley J, Rademacher DJ, Stancati JA, Sisson KA, Sortwell CE, Paumier KL, Gebremedhin KG, SteeceCollier K (2015) Interrogating the aged striatum: Robust survival of grafted dopamine neurons in aging rats produces inferior behavioral recovery and evidence of impaired integration. Neurobiol Dis 77, 191-203.

[29] Grealish S, Heuer A, Cardoso T, Kirkeby A, Jonsson M, Johansson J, Bjorklund A, Jakobsson J, Parmar M (2015) Monosynaptic tracing using modified rabies virus reveals early and extensive circuit integration of human embryonic stem cell-derived neurons. Stem Cell Rep 4, 975-983.

[30] Adler AF, Cardoso T, Nolbrant S, Mattsson B, Hoban DB, Jarl U, Wahlestedt JN, Grealish S, Bjorklund A, Parmar M (2019) hESC-derived dopaminergic transplants integrate into basal ganglia circuitry in a preclinical model of Parkinson's disease. Cell Rep 28, 3462-3473.e3465.

[31] Haque NS, LeBlanc CJ, Isacson O (1997) Differential dissection of the rat E16 ventral mesencephalon and survival and reinnervation of the 6-OHDA-lesioned striatum by a subset of aldehyde dehydrogenase-positive $\mathrm{TH}$ neurons. Cell Transplant 6, 239-248.

[32] Grealish S, Jonsson ME, Li M, Kirik D, Bjorklund A, Thompson LH (2010) The A9 dopamine neuron component in grafts of ventral mesencephalon is an important determinant for recovery of motor function in a rat model of Parkinson's disease. Brain 133, 482-495.
[33] Hintiryan H, Foster NN, Bowman I, Bay M, Song MY, Gou L, Yamashita S, Bienkowski MS, Zingg B, Zhu M, Yang XW, Shih JC, Toga AW, Dong HW (2016) The mouse cortico-striatal projectome. Nat Neurosci 19, 1100-1114.

[34] Fisher BE, Petzinger GM, Nixon K, Hogg E, Bremmer S, Meshul CK, Jakowec MW (2004) Exercise-induced behavioral recovery and neuroplasticity in the 1-methyl-4phenyl-1, 2, 3, 6-tetrahydropyridine-lesioned mouse basal ganglia. J Neurosci Res 77, 378-390.

[35] VanLeeuwen JE, Petzinger GM, Walsh JP, Akopian GK, Vuckovic M, Jakowec MW (2010) Altered AMPA receptor expression with treadmill exercise in the 1-methyl-4phenyl-1,2,3,6-tetrahydropyridine-lesioned mouse model of basal ganglia injury. $J$ Neurosci Res 88, 650-668.

[36] Shin M-S, Jeong H-Y, An D-I, Lee H-Y, Sung Y-H (2016) Treadmill exercise facilitates synaptic plasticity on dopaminergic neurons and fibers in the mouse model with Parkinson's disease. Neurosci Lett 621, 28-33.

[37] Steinbeck JA, Choi SJ, Mrejeru A, Ganat Y, Deisseroth K, Sulzer D, Mosharov EV, Studer L (2015) Optogenetics enables functional analysis of human embryonic stem cellderived grafts in a Parkinson's disease model. Nat Biotech 33, 204-209.

[38] Hicks AU, Hewlett K, Windle V, Chernenko G, Ploughman M, Jolkkonen J, Weiss S, Corbett D (2007) Enriched environment enhances transplanted subventricular zone stem cell migration and functional recovery after stroke. Neuroscience 146, 31-40.

[39] Brundin P, Karlsson J, Emgard M, Schierle GS, Hansson O, Petersen A, Castilho RF (2000) Improving the survival of grafted dopaminergic neurons: A review over current approaches. Cell Transplant 9, 179-195.

[40] Hagell P, Brundin P (2001) Cell survival and clinical outcome following intrastriatal transplantation in Parkinson disease. J Neuropathol Exp Neurol 60, 741-752.

[41] Cohen AD, Tillerson JL, Smith AD, Schallert T, Zigmond MJ (2003) Neuroprotective effects of prior limb use in 6hydroxydopamine-treated rats: Possible role of GDNF. $J$ Neurochem 85, 299-305.

[42] Tajiri N, Yasuhara T, Shingo T, Kondo A, Yuan W, Kadota T, Wang F, Baba T, Tayra JT, Morimoto T (2010) Exercise exerts neuroprotective effects on Parkinson's disease model of rats. Brain Res 1310, 200-207.

[43] Real CC, Ferreira AF, Chaves-Kirsten GP, Torrao AS, Pires RS, Britto LR (2013) BDNF receptor blockade hinders the beneficial effects of exercise in a rat model of Parkinson's disease. Neuroscience 237, 118-129. 